\title{
Local Hardness Variation of Ti50Cu32Ni15Sn3 Processed by Laser Beam Melting (LBM)
}

\author{
http://dx.doi.org/10.3991/ijes.v3i1.4293 \\ M. Karg, B. Ahuja, O. Hentschel and M. Schmidt \\ Friedrich-Alexander-Universität Erlangen-Nürnberg (FAU), Germany
}

\begin{abstract}
Amorphous metals which are synonymously called metallic glasses form a rather young group of engineering materials. Compared to crystalline metals they offer unique combinations of properties: tensile strength, hardness, elastic strain, resistance against corrosion and abrasive wear are rather high. In order to minimize crystal growth, rapid solidification from the liquid phase is required. High cooling rates are a characteristic property of the additive manufacturing technology Laser Beam Melting in Powder Bed (LBM). This paper shows first results of processing Ti50Cu32Ni15Sn3 by LBM. Unlike many other alloys with high glass forming ability, it does not contain costly rare earth elements. No literature is known to the authors about LBM of this material. Because relative density close to $\mathbf{1 0 0}$ $\%$ is a prerequisite for producing parts with high mechanical performance, a parameter study was conducted varying scan speed, hatch distance and laser power in wide ranges. The obtained samples are characterized by metallographic sections, hardness measurements and X-ray diffraction. Apart from reaching high relative densities, a wide variation in Vickers hardness over the length of samples was found. It corresponds to the locally different thermodynamic conditions. Apart from introducing a new material with promising properties to the manufacturing technology of LBM, this might open up a new approach to modify mechanical material properties in a single work piece made from uniform powder by adapting LBM process parameters. Both the range of applications for $\mathrm{LBM}$ as well as the range of geometries producible from amorphous metals might be expanded.
\end{abstract}

Index Terms-Additive Manufacturing, Amorphous Metal, Laser Beam Melting, Pyrometer, Ti-Cu-Ni-Sn

\section{INTRODUCTION}

The development of metallic glass goes back to 1960 when P. Duwez et al. achieved amorphous metal by rapid quenching of liquid Au75Si25 [1]. Since then, numerous alloys have been researched for their glass forming abilities, usually being composed of three or more chemical elements. The cooling rates required for glass formation, also called vitrification, depend strongly on alloy composition and can be as low as $0,1 \mathrm{~K} / \mathrm{s}$ for Palladium-based alloys. Amorphous metals based on Zirconium have already been commercialized. Such alloying components are either costly on their own (e.g. Pd) or because of their strong chemical attraction to Oxygen (e.g. Zr) which requires special precautions for processing.

Technologies to produce bulk metallic glasses are e.g.: Oxide-fluxing, Melt spinning, Mechanical alloying, Vapour condensation, Diffusion induced amorphization in multilayers, Ion beam mixing, Hydrogen absorption, Inverse melting or injection casting. All suffer from severe restrictions to create complex geometries as well as provide sufficient cooling rates for large parts. In this context, dimensions over $1 \mathrm{~mm}$ are considered large. Manufacturing such a material additively through LBM might offer higher geometric freedom.

As a compromise between material cost, handling efforts and glass forming ability, the alloy Ti50Cu32Ni15Sn3 was chosen for these experiments. According to $[2,3,4,5,6]$, it offers interesting mechanical properties in compression testing such as an elastic strain limit of $1.8 \%$, a plastic deformation of $4 \%$ and compressive strength of about $2000 \mathrm{MPa}$. This gives it an impressive strength to weight ratio and potential for lightweight structures. According to [7] required minimum cooling rate should be in the order of $10^{2} \mathrm{~K} / \mathrm{s}$, which could be reachable in the LBM process. The critical cooling rat is calculated from (1) and (2) according to [7].

$$
\begin{gathered}
\log R_{c}=14.99-19.441 \gamma_{m} \\
\gamma_{m}=\frac{2 T_{x}-T_{g}}{T_{l}}
\end{gathered}
$$

$$
\begin{aligned}
& \mathrm{Y}_{\mathrm{m}}: \text { Glass forming ability criterion } \\
& \mathrm{T}_{\mathrm{x}} \text { : Onset crystallisation temperature } \\
& \mathrm{T}_{\mathrm{g}}: \text { Glass transition temperature } \\
& \mathrm{T}_{\mathrm{l}} \text { : Liquidus temperature } \\
& \mathrm{R}_{\mathrm{c}} \text { : Critical cooling rate }
\end{aligned}
$$

Pauly et al. have recently published experimental results from LBM of an iron-based amorphous metal, yet with the main focus on showing the feasibility in principle [8].

\section{MATERIALS AND METHODS}

\section{A. Powder Material}

The Ti50Cu32Ni15Sn3 powder used for the experiments was atomized in an Argon atmosphere by Nanoval $\mathrm{GmbH}$, Berlin. Results of the suppliers's particle size distribution measurement are shown in Figure 1. To ensure homogeneous powder layer recoating in LBM, the fraction from $20-50 \mu \mathrm{m}$ was separated by vibration sieving under inert atmosphere. Only the sieved powder was used for LBM experiments. For qualitative assessment of the particle sizes, scanning electron microscopy (SEM) was employed. 


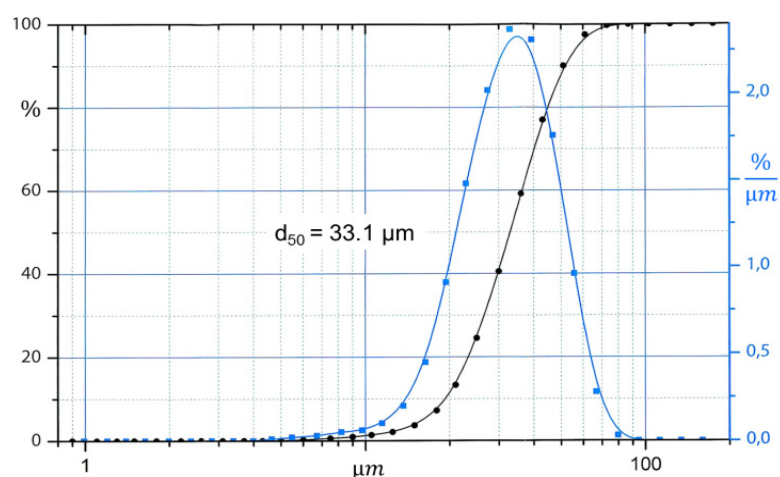

Figure 1. Particle size distribution measured by powder supplier [9]

\section{B. LBM Processing}

An SLM 50 LBM machine from the manufacturer ReaLizer $\mathrm{GmbH}$ is used for the experiments. It features a rotational recoater with a flexible silicone rubber lip in contact with the powder and a single-mode $\mathrm{Yb}$ :YAG fiber laser with a wavelength of $1070 \mathrm{~nm}$ and a maximum output power of $100 \mathrm{~W}$. The manufacturer specifies a minimum focus diameter of $10 \mu \mathrm{m}$. Build platforms made of $99.5 \%$ pure aluminium are used because of their high heat conductivity. To ensure homogeneous powder coating and sufficient connection of the first molten layer, the build platforms were blasted with corundum $\left(\mathrm{Al}_{2} \mathrm{O}_{3}\right)$ particles driven by pressurized air. The process chamber is flushed with recirculating and filtered Argon.

The practical process of LBM is approached step by step with increasing complexity. Firstly, thin walls built from vertically overlapping single melt tracks are produced and analyzed, secondly cuboids on the platform. No contour scanning and no effort to optimize surface roughness is made, as it is of secondary interest for this research. The layer thickness is constant at $30 \mu \mathrm{m}$ and the platform not heated.

Laser power, hatch distance and scan speed are varied. As there are no previous results from literature that could give an orientation for this alloy, a wide range of line energies is covered, as shown in Figure 3. For Laser Power, values of $40 \mathrm{~W}, 60 \mathrm{~W}, 70 \mathrm{~W}, 80 \mathrm{~W}$ and $100 \mathrm{~W}$, for Scan speeds, 10 different values between minimum 40 $\mathrm{mm} / \mathrm{s}$ and $800 \mathrm{~mm} / \mathrm{s}$ are used. Hatch distances of $20 \mu \mathrm{m}$ and $50 \mu \mathrm{m}$ are used. Figure 2 gives an overview over the 50 parameter combinations used for thin wall experiments. Figure 3 shows the Line energies resulting from these values in a logarithmic scaling. For stronger statistics, 5 thin walls of each parameter combination with 5 $\mathrm{mm}$ length each are built. After visual inspection of the results, the parameters which have led to the most homogenous thin walls are selected for choosing parameters for the cuboid samples in the next step.

For the production of cubic samples with an edge length of $5 \mathrm{~mm}$, the scan direction is alternated by $90^{\circ}$ parallel to the $\mathrm{X}$ and $\mathrm{Y}$ axis of the machine coordinate system from layer to layer - so called alternating scanning. The cuboid samples are rotated $45^{\circ}$ around the vertical $\mathrm{z}$-axis of the machine coordinate system, as can be seen in Figure 5. As a result, scan vectors for hatching vary in length from almost $0 \mathrm{~mm}$ in the corners of a cube like in Figure 6, up to $7 \mathrm{~mm}$ in the diagonal. No more complex scan strategies such as e. g. stripes or chessboard patterns are used. Figure 5 shows the arrangement of cuboid samples on the platform.

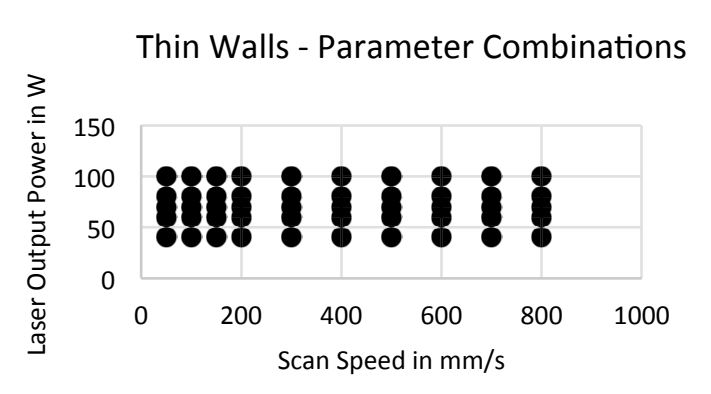

Figure 2. Investigated parameter field of scan speeds and laser power

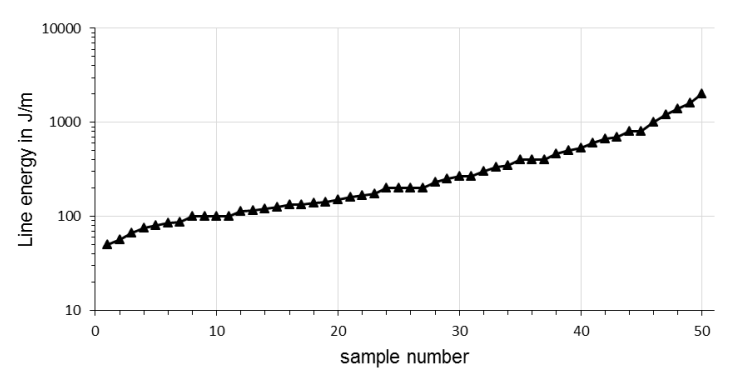

Figure 3. Line energy for the thin walls in a logarithmic scale

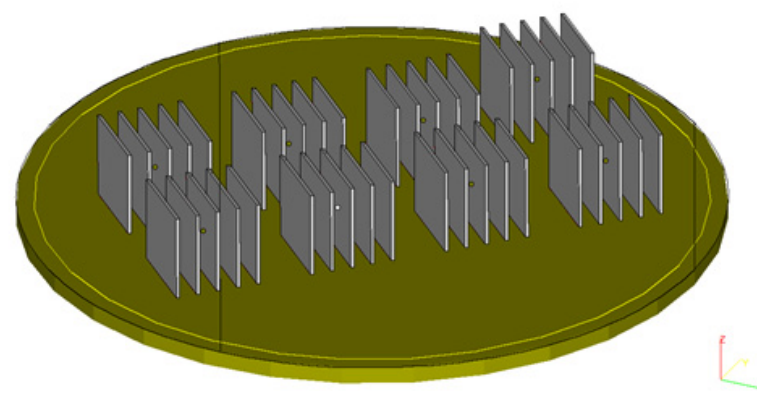

Figure 4. Arrangement of $5 \mathrm{~mm}$ long thin walls on the build platform

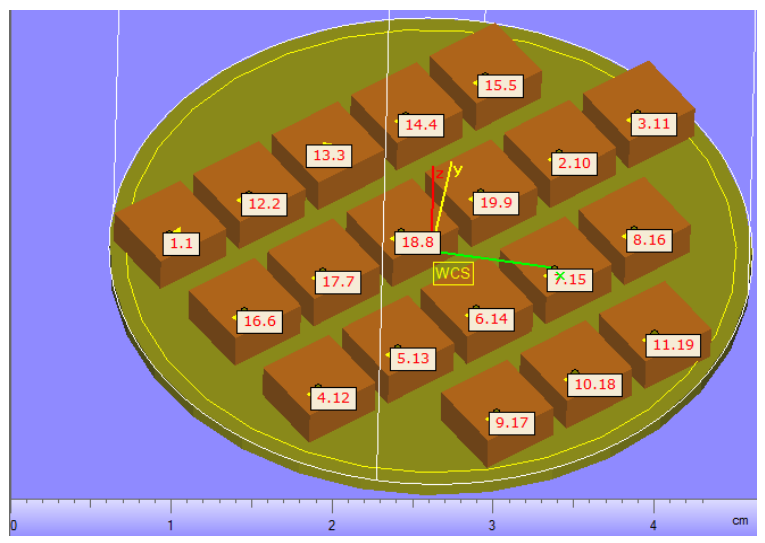

Figure 5. Arrangement of $5 \mathrm{~mm}$-cuboid samples on the build platform

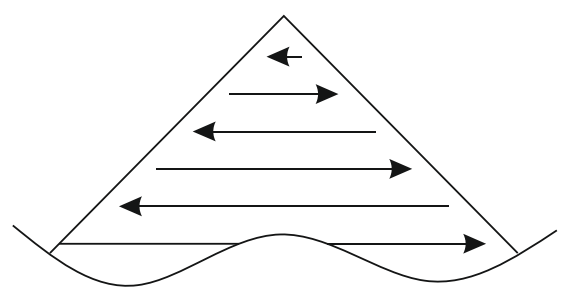

Figure 6. Scan vectors rotated 45 degrees to cube edges 


\section{Analysis}

For thermal analysis, a high speed pyrometer IMPAC IMGA 740-LO from the manufacturer LumaSense Technology $\mathrm{GmbH}$ is used. It offers a sampling rate of 100 $\mathrm{kHz}$. According to the equipment data sheet, the InGaAs detector is sensitive in a range from $200{ }^{\circ} \mathrm{C}$ to $1000{ }^{\circ} \mathrm{C}$, corresponding to a spectral range from $1.58 \mu \mathrm{m}$ to $2.2 \mu \mathrm{m}$ of heat radiation wave length. The pyrometer optics are set up laterally, that means a stationary spot is observed. During LBM, the focus of the processing laser repeatedly passes through the measurement spot of the pyrometer. This spot is of elliptical shape due to the angled mounting position of the pyrometer optics, the two axes being 0.5 and $0.6 \mathrm{~mm}$ long. Because of the heterogeneous nature of the material in the measurement spot - partially loose powder, melt as well as resolidified metal - the true effective emissivity is not easy to determine. Emissivity does not only depend on temperature and state of aggregation, but also on surface roughness. In this paper, the emissivity is approximated as a constant value of 1 .

For etching the metallographic samples, the following etachant is used: $1000 \mathrm{ml} \mathrm{H}_{2} \mathrm{O}, 8 \mathrm{ml} \mathrm{HNO}_{3}, 2.5 \mathrm{ml} \mathrm{HCl}$ and $7.5 \mathrm{ml} \mathrm{HF}$.

Vickers hardness is measured with a Fischerscope H100VP device and the corresponding control software Win HCU. A force of $500 \mathrm{mN}$ is built up over a ramp of $20 \mathrm{~s}$ duration, held stable for $5 \mathrm{~s}$ and released again over $20 \mathrm{~s}$.

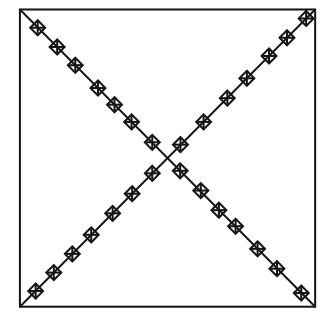

Figure 7. location of hardness measurements on horizontal microsection

As shown in Figure 7, hardness measurememts are conducted along the diagonals of the horizontal cross sections of cuboid samples.

For X-Ray diffraction (XRD), a Seifert Stress-Analyzer XRD 3003 device is used. The detection angle is varied from $20^{\circ}$ to $162^{\circ}$ in steps of $0.02^{\circ}$. The Diameter of the XRD measurement spot is $1 \mathrm{~mm}$. The samples for XRD measurements have been grinded and polished in the same way as those for metallographic etching.

\section{RESULTS AND DISCUSSION}

\section{A. Powder Characterization}

The sieved powder particles are analyzed in SEM, as shown in Figure 5. The particles appear mostly spherical in shape with smooth surfaces. Only few particles smaller than $20 \mu \mathrm{m}$ are detected, indicating the desired effect of sieving. Satellite particles are also rare, so no detrimental effects on the powder layer recoating is to be expected.

\section{B. Thin Walls}

Most homogeneous thin walls have been produced with line energies from $466 \mathrm{~J} / \mathrm{m}$ to $800 \mathrm{~J} / \mathrm{m}$. An example of a platform with thin walls of different quality is shown in Figure 9.

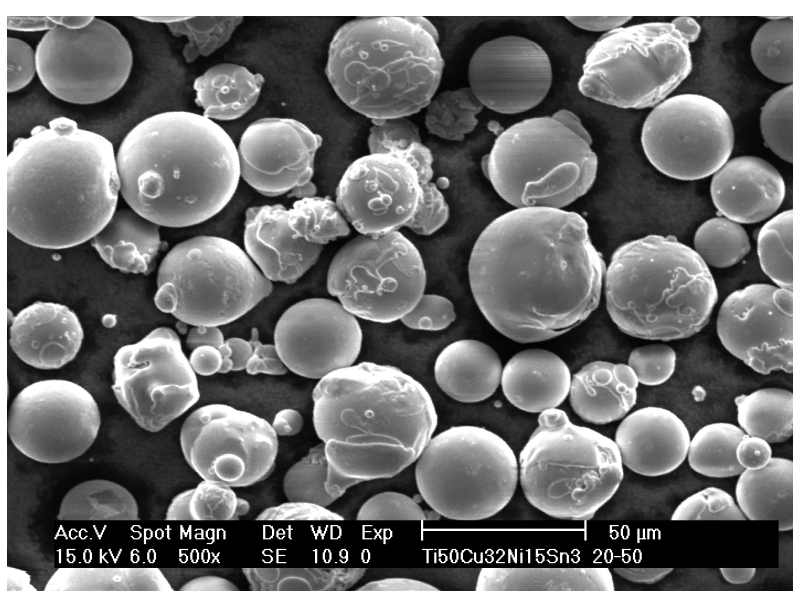

Figure 8. SEM picture of sieved powder used for LBM

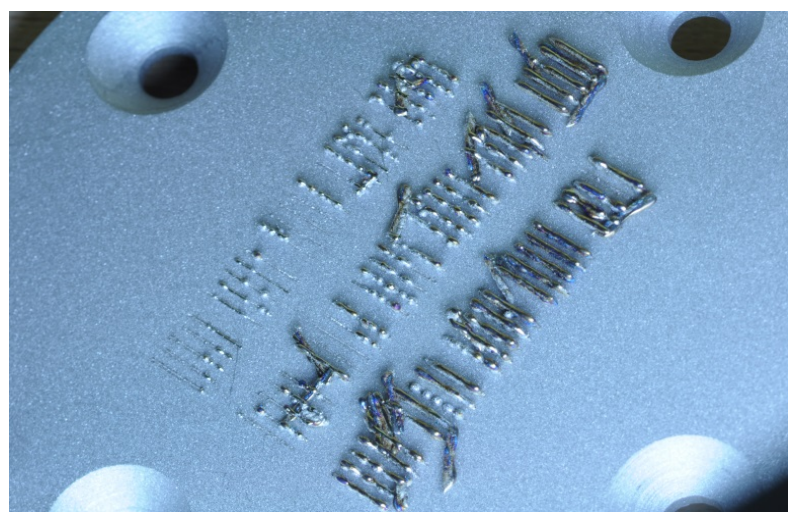

Figure 9. Thin walls of $5 \mathrm{~mm}$ length on the platform

For building cubes, scan speeds between 100 and $500 \mathrm{~mm} / \mathrm{s}$, laser powers between $60 \mathrm{~W}$ and $100 \mathrm{~W}$ and hatch distances of $25 \mu \mathrm{m}$ and $50 \mu \mathrm{m}$ are used.

\section{Cubes}

During building cubes on platform, a strong tendency towards balling is observed with many parameter combinations. The respective cuboid samples could not be continued after a few layers because the recoater got stuck there. The balling effect is most prominent in the corners of the cuboids. During the LBM process, an afterglow of these corner areas could be observed with the naked eye, indicating a strong heat accumulation. The best produced samples are shown in Figure 10.

The square cross-sections of the samples had been hatched not in parallel to the sample edges, but under a $45^{\circ}$ angle. As an effect, scan vector length differs from a maximum of $7 \mathrm{~mm}$ in the diagonal to a minimum of close to $0 \mathrm{~mm}$ in the corners.

Figure 11 and 12 show etched horizontal microsections of cuboids, magnified from the center and the corner of a sample, respectively. Apart from some pores and cracks, relative density seems to be close to $100 \%$, which is a necessary requirement for high strength mechanical parts. The scan pattern with an angle of $45^{\circ}$ towards the cuboids edges is easily found in the etchings. The corners of the cuboids look fundamentally different to the center areas: Here, no melt tracks can be identified, the porosity is higher and the microstructure seems to be nanocrystalline, appearing grey in Figure 12. A similar microstructure can be observed on the borders of overlapping melt tracks. 
PAPER

LOCAL HARDNESS VARIATION OF Ti50CU32Ni15SN3 PROCESSED BY LASER BEAM MELTiNG (LBM)

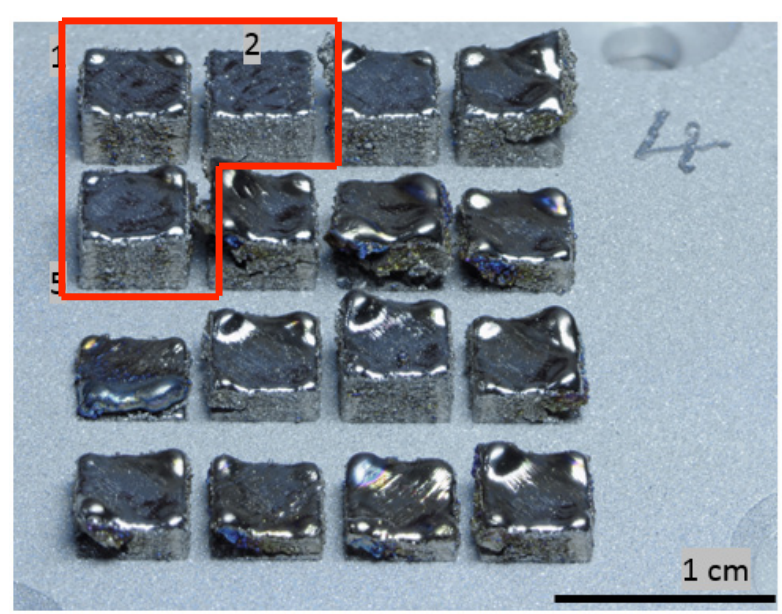

Figure 10. Best results in cubes on platform highlighted in the red frame, marked 1, 2 and 5

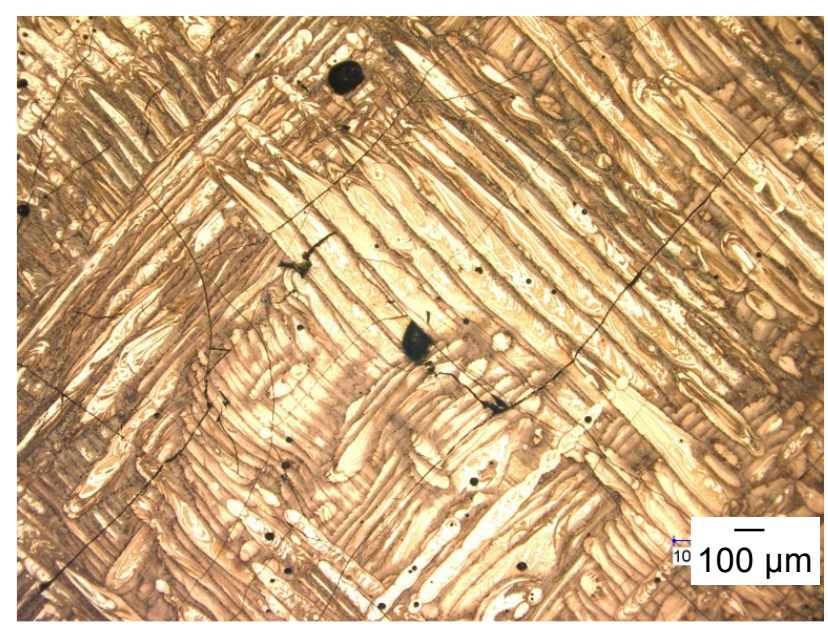

Figure 11. Etched horizontal microsection, center of sample

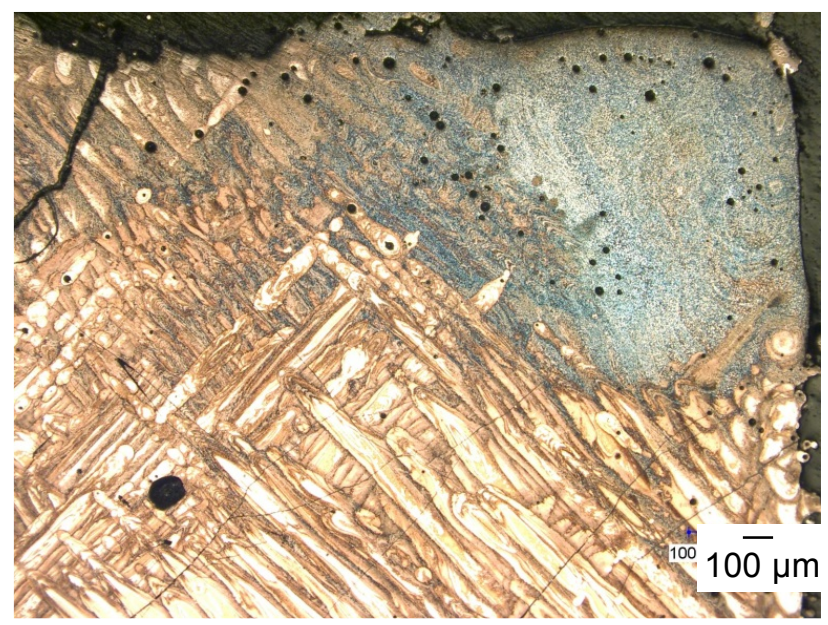

Figure 12. Etched horizontal microsection, corner of sample

\section{X-Ray diffraction}

The results from x-ray diffraction measurement in the center of a polished cuboid sample are shown in Figure 13. Sharp peaks can be found which indicate crystallinity [8]. Because of a lack of comparison data for the specific alloy processed here, no further statement is possible.

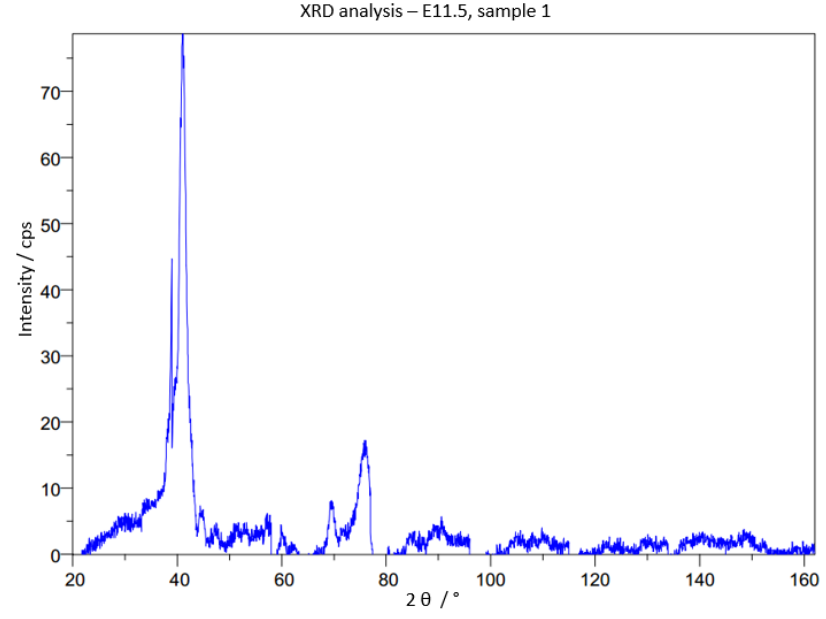

Figure 13. X-ray diffraction spectrum

\section{E. Hardness Measurement}

Vickers hardness measured from three different cubes manufactured with different LBM parameters shows a consistent course depending on the distance from the samples' center. In the corners of the samples, average hardness is below $400 \mathrm{HV}$. In the center, average hardness is about $700 \mathrm{HV}$. This might be caused by a lower crystallinity in the center. The values in the samples' center might correspond to high strength.

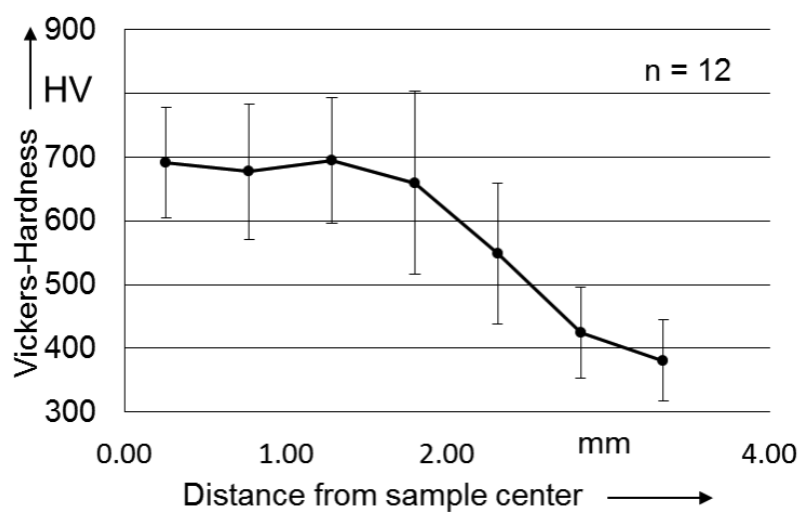

Figure 14. Hardness measured at horizontal microsection

\section{F. Pyrometer Analysis}

The observation of an afterglow in the corners of cuboid samples is confirmed by the results of pyrometer measurements shown in Figure 15 and 16: the temperature in the corner is much higher than in the center of samples, building up over multiple passings of the laser focus. Each time the laser focus passes through the pyrometer spot, it causes a sharp peak in the radiation temperature. The interval between two consecutive passings becomes shorter over time due to the $45^{\circ}$ rotation of the hatch vectors. Also the geometry is expected to have an influence on the heat conditions: the loose powder surrounding the corner should act as a thermal insulator compared to the solidified metal in the samples' center. 


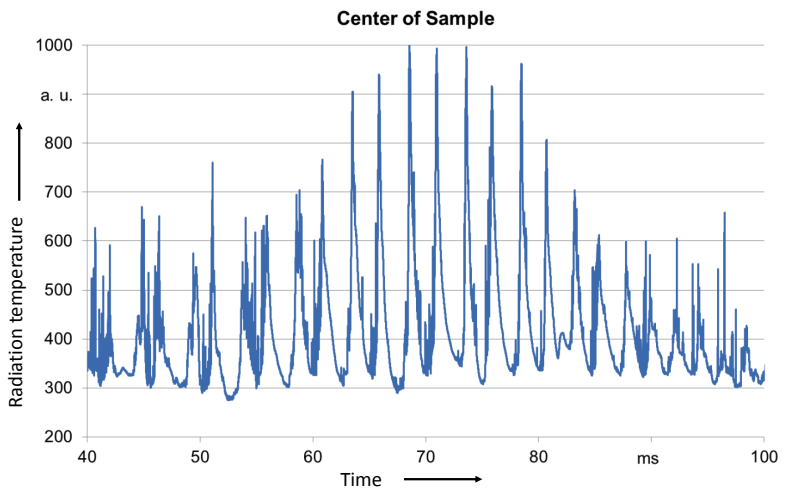

Figure 15. Radiation temperature measured in the center of a sample cube during LBM build-up

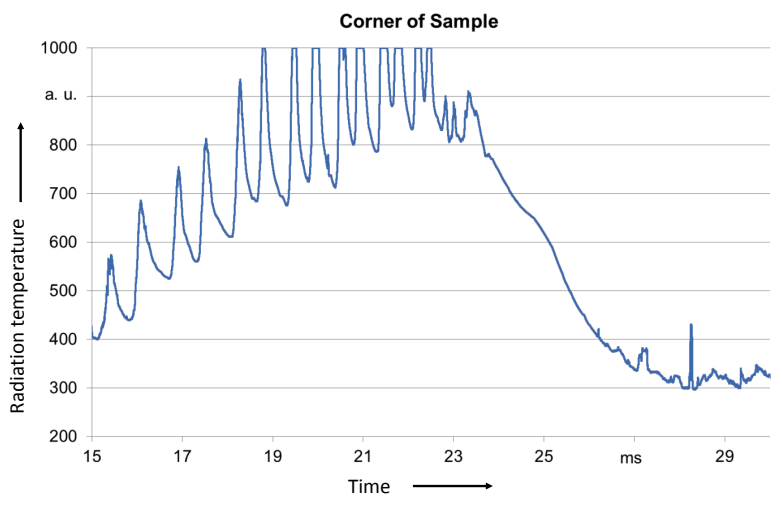

Figure 16. Radiation temperature measured in the corner of a sample build up under identical conditions as in Figure 15

\section{CONCLUSION AND OUTLOOK}

By conducting parameter studies for processing Ti50Cu32Ni15Sn3 powder in Laser Beam Melting (LBM), it is proven that cuboid samples with $5 \mathrm{~mm}$ edge length can be built with relative density close to $100 \%$. Etched Microsections give a very much different visual impression in the corners of samples compared to their center. They can be interpreted as higher crystallinity in the corners. The XRD analysis implies crystallinity in the samples' centers, too, by well-defined peaks in the spectrum. Further interpretation is prohibited by lack of XRD reference data for this specific alloy. Hardness measurements show promising values around $700 \mathrm{HV}$ in the center of the samples, declining towards $400 \mathrm{HV}$ in the corners of the samples. This correlates with the different cooling conditions due to the geometry and the applied scan strategy: Cooling rates in the center should be increased by the surrounding resolidified metal compared to the corners with more surrounding powder. The powder bed has a lower heat conductivity than solid metal.

This might be used in the future to intentionally modify mechanical properties of structural parts built by LBM of TiCuNiSn alloys with adapted scan strategies. Research is ongoing to distinguish effects of the sample geometry from those of scanning strategy.

An issue in the produced samples that is not acceptable for mechanically strong parts is the occurrence of cracks and pores. This must be adressed in future research in order to build Mechanical characterization is in tensile and compressive tests is to be performed in the next step.

\section{ACKNOWLEDGMENT}

We acknowledge the work performed by Fabian Wegler as part of his Bachelor's Thesis and Laura Rösch in her Master's Thesis, both tutored by the authors at the Institute of Photonic Technologies (LPT).

\section{REFERENCES}

[1] W. Klement, R. Willens, P. Duwez,, "Non-crystalline Structure in Solidified Gold-Silicon Alloys", in Nature 1960 http://dx.doi.org/10.1038/187869b0

[2] A. Inoue, "High Strength Bulk Amorphous Alloys with Low Critical Cooling Rates", in Material Transactions, JIM 36(7): 866-875, 1995

[3] T. Zhang, A. Inoue, "Thermal and Mechanical Properties of Ti-Ni$\mathrm{Cu}-\mathrm{Sn}$ Amorphous Alloys with a Wide Supercooled Liquid Region before Crystallization", in Materials Transactions, JIM, Vol. 39, No. 10 pp. 1001-1006, 1998

[4] C. Ma, H. Soejima, S. Ishihara, K. Amiya, N. Nishiyama, A. Inoue, "New Ti-Based Bulk Glassy Alloys with High GlassForming Ability and Superior Mechanical Properties", in Materials Transactions. 2004 http://dx.doi.org/10.2320/matertrans. $\underline{45.3223}$

[5] Y. Kim, W. Kim, D. Kim, "A development of Ti-based bulk metallic glass", in Materials Science and Engineering A, 2004 http://dx.doi.org/10.1016/j.msea.2003.10.115

[6] J. Löffler, „Bulk metallic glasses“, in Intermetallics. 2003 http://dx.doi.org/10.1016/S0966-9795(03)00046-3

[7] X. Du, J. Huang, C. Liu, Z. Lu, "New criterion of glass forming ability for bulk metallic glasses", in J. Appl. Phys. 2007 http://dx.doi.org/10.1063/1.2718286

[8] S. Pauly, L. Löber, R. Petters, M. Stoica, S. Scudino, U. Kühn, J. Eckert, "Processing metallic glasses by selective laser melting", in Materials Today, Volume 16, Numbers 1/2, 2013

[9] Nanoval GmbH \& Co. KG, product certificate, 2014

\section{AUTHORS}

M. Karg, B. Ahuja, O. Hentschel and M. Schmidt are with the Institute of Photonic Technologies (LPT), Friedrich-Alexander-Universität Erlangen-Nürnberg (FAU), Germany.

This research was conducted within the Collaborative Research Center CRC 814 Additive Manufacturing - "SFB 814 Additive Fertigung"subproject A 5 "Selective Laser Melting of Composite Material Systems for the Manufacture of Metallic Light-Weight Structures" and supported by Erlangen Graduate School in Advanced Optical Technologies (SAOT) in the framework of the German excellence initiative. Both SFB 814 and SAOT receive financial support from the German Research Foundation DFG. This article is an extended and modified version of a paper presented at the International Conference on Additive Technologies (ICAT2014), held from 15-17 October 2014 in Vienna, Austria. Submitted 30 November 2014. Published as resubmitted by the authors 10 March 2015. 